\title{
THE PROGRESS OF DEVELOPMENT OF FOUR KICKERS' BUMP SYSTEM FOR INJECTION IN HLS RING
}

\author{
$\underline{\text { X.Q. Wang }}{ }^{\#}$, Y.J. Pei, L. Shang, L. Wang, K.J. Fan, H.L. Xu, D.M. Jiang \\ Y.B. Leng, G.C. Wang, X.Y. He, F. Zhao, J.G. Zhang \\ NSRL, USTC, P.O. Box. 6022, Hefei, Anhui 230029, China
}

\section{Abstract}

There are three kicker magnets to manipulate local bump orbit in Hefei storage ring, which could satisfy basically the commissioning and operations of General Purpose Light Source (GPLS) mode. In the Phase Two Project of HLS, four kicker magnets will be employed and installed in one straight section to form a ladder-shaped bump orbit. So the parameters of bump orbit are independent of the ring lattice. The new system also satisfies the multicycle and multiturn injection of High Brightness Light Source (HBLS) mode and other modes, and it expected to run stably for a long time. This paper presents the development of the new bump orbit system.

\section{INTRODUCTION}

A multicycle and multiturn injection scheme is adopted to accumulate beam in HLS ring ${ }^{1}$. The injection system consists of three kicker magnets, one pulse septum magnet, one DC septum magnet and their power supplies ${ }^{2}$. The pulse septum magnet which is made of steel sheet and copper current strip with $\mathrm{Al}_{2} \mathrm{O}_{3}$ powder coating runs well for ten years, and its steel sheets have some absorption capacity after baking ${ }^{3}$. The air-core of kicker magnet in the vacuum pipe produces pulse field. The pulse formation circuit uses a spark gap switch to generate a half sinewave current pulse with attenuation which can satisfy the commissioning and operation of the GPLS mode ${ }^{4-6}$. Fig.1 shows the distribution of the magnets and vacuum chambers. Fig.2 shows local bump orbits corresponding separately to the GPLS with emittance of $133 \mathrm{~nm} \cdot \mathrm{rad}$ and the HBLS-1 of $27 \mathrm{~nm} \cdot \mathrm{rad}$.

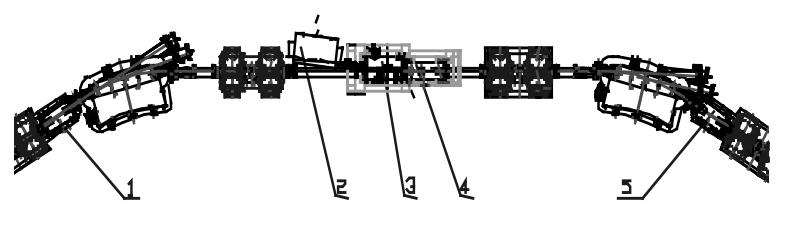

1.4.5: kicker; 2: DC septum; 3: pulse septum.

Fig.1 Layout of the injection system of the HLS ring

There are four quadrupole magnets and two bending magnets within the bump orbit of three kicker magnets. So the parameters of kicker magnets are decided by local Twiss functions and phase shifts. Such bump orbit system

\footnotetext{
\# Email: wangxaqi@ustc.edu.cn
}

couldn't satisfy injection ${ }^{2,7,8}$ of HBLS-2 mode of $13 \mathrm{~nm} \cdot \mathrm{rad}$. The two kickers (first and fifth in fig.1) occupy installation space suitable for two sextuple magnets, it's a disadvantage and decreases the dynamic aperture.

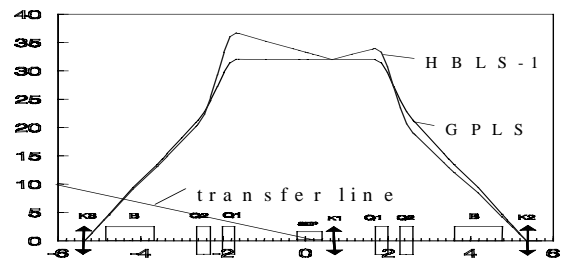

Fig. 2 Scheme of bump orbits and the layout of the inj. sys. In order to form a reasonable bump orbit, HBLS-1 requires the middle kicker to deflect beam with a small angle. However, it is too difficult for the pulse power supply with spark-gap switches to generate such a small pulse current with little time jitter, furthermore, the lifetime of switch is too short and needs maintenance frequently.

In the new injection system, four kicker magnets will be installed in one straight section, and thyratrons will be used as switch, which can decrease the time jitter to less than 10ns. Two sextupole magnets will be added at the place which were now occupied by two kickers, this will increase the dynamic aperture.

\section{NEW OVERALL ARRANGEMENT}

The HLS straight section for injection is only 3 meters long, it's quite difficult to install four kickers in such a short extent. Installation method and injection tracking ${ }^{8}$ have been carefully studied. The overall arrangement of the new bump orbit system is shown in fig.3. Fig.4 shows the redesigned distribution of ring.

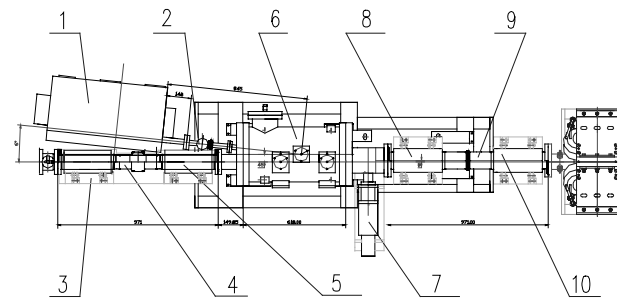

1: DC septum; 2, 7: beam monitor; 3, 5, 8, 10: kicker; 6: pulse septum; 4, 9: ceramic vacuum.

Fig.3 Plane distribution of magnets and scheme of bump orbit in the inj. sys. with centralizing overall arrangement. 


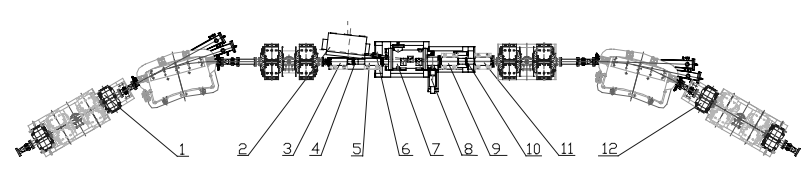

Fig.4 New plane distribution diagram of the area involved in injection system reconstruction of HLS ring.

\section{PREFABRICATED STUDY}

Some key techniques have been studied and solved since 1993.

\subsection{Kicker magnets}

Kickers with $270 \mathrm{~mm}$ long are divided into two types in cross section ${ }^{11}$. A pair of kickers (shown in fig.3) are installed upstream the pulse septum, the others are installed downstream. Fig. 5 shows the cross section of a kind of yoke which size is $92 \times 36 \mathrm{~mm}^{2}$, the other is $108 \times 36$ $\mathrm{mm}^{2}$. The material of yoke is MnZn ferrite which has a wide frequency spectrum ${ }^{12}$. A prototype kicker magnet with the ferrite yoke has been assembled to study the properties of the ferrite and the distribution of magnetic field. Fig. 6 shows relationship between the magnetic field and the excited current $(\mathrm{B} / \mathrm{I})$, the measured maximum magnetic field is about 2.9 kilo gauss which far exceed the need of injection. The $\mathrm{B} / \mathrm{I}$ curve (signed by black dots in fig.6) is approximately linear quite conform the calculated result

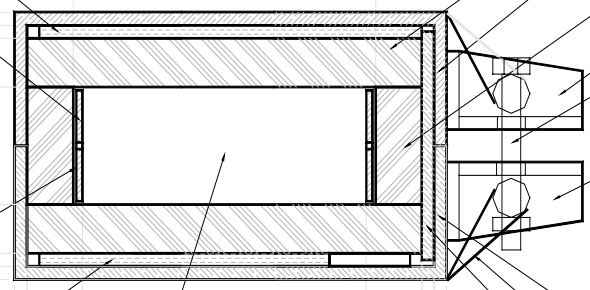

Fig.5 Cutaway view of kicker measured \& calculated

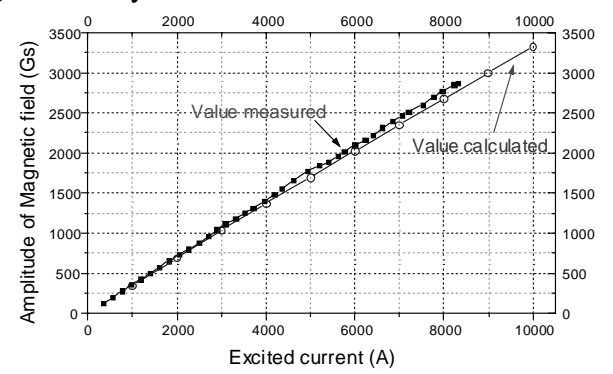

Fig. $6 \mathrm{~B}_{\mathrm{P}} / \mathrm{I}_{\mathrm{P}}$ Curves measured \& calculated

\subsection{Pulse power supply}

Two pulse power supplies are employed to drive kickers separately, one is for $\mathrm{K} 1$ and $\mathrm{K} 2$, the other is for $\mathrm{K} 3$ and $\mathrm{K} 4$, this can decrease the timing jitter among kickers. Thyratron, EEV CX1174, is used as main switch, which has a good property with timing jitter less than $5 \mathrm{~ns}$ and the stability up to $0.1 \%^{14,15}$. A prototype pulse power supply has been constructed, some new techniques have been adopted to improve its performance ${ }^{14,15}$.
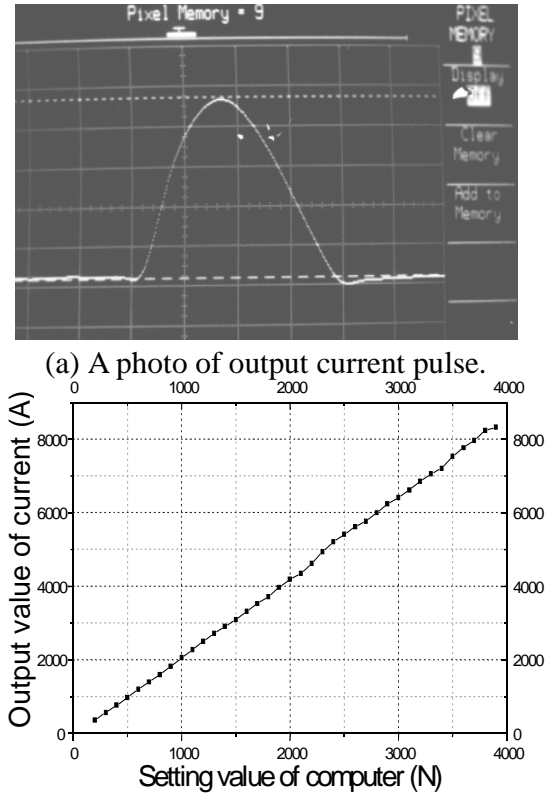

(b) The pertinence of output current \& control number.

Fig.7 The prototype of pulse power supply of kicker magnet.

\subsection{Ceramic vacuum chamber}

The ceramic chambers are both $971 \mathrm{~mm}$ long (No.3 and No.9 in fig.3), their inner aperture are $80 \times 24 \mathrm{~mm}^{2}$ and $96 \times 24 \mathrm{~mm}^{2}$ respectively, the cross section is racetrack. They are key hardware and are being prefabricated now. Physical analysis indicates ${ }^{16}$ that coatings with suitable metal material in inner surface with proper thickness can meet requirements of vacuum impedance and pulse magnetic penetrability. The losing impedance measurement system, based on HP instruments and HPVEE software, is set up ${ }^{17}$. The techniques related to fabrication of ceramic chamber are studied.

\subsection{Magnetic field measurement}

Surveying the electromagnetic characteristic of the yoke, adjusting the waveform of kickers' field and measuring the distribution of magnetic field, will be done soon. Automatic measurement system of micro second magnetic field has been set up. Magnetic field distribution of prototype kicker ${ }^{18}$ was obtained. The measurement data (see in fig.6 and fig.7) indicates that it is feasible using this kind of ferrite material to meet the requirement of injection.

\section{CONSTRUCTION DESIGN}

Since June of 1997, the project came into the phase of construction. Some contracts have been signed. All the non-standard measurement facilities are being constructed. Parts of them have already finished by the end of 1998 . Four vacuum chambers with DC clearing electrodes and close orbit monitors will be installed in the ring. These new increased electrodes will improve symmetry of the ring lattice ${ }^{19}$ and restrain ion trapping instability ${ }^{20}$. 


\section{REFERENCE}

[1] Y J Pei, Multiturn injection, Reported in particle dynamic symp. (Huangshan, China), 1981.

[2] X Q Wang et al., Proce. of Hefei ICSRL (Hefei, China), 1989, p.192.

[3] Y J Pei et al., Nucl. Instr. and Meth. in Phys. Res. A 398 (1997) 368.

[4] X Q Wang et al., Proc. of 1st Taiwan Straits Symp. on SR, 1993, p.32.

[5] X Q Wang et al., Jour. of China Uni. of S. \& T., 23(2) (1993) 151.

[6] X Q Wang, Jour. of China Univ. of S. \& T. 24(4) (1994) 536.

[7] X Q Wang et al., 6th China-Japan joint symp. on acc. for nucl. S. and Their Appl., 1996, p.300.

[8] L Shang et al., Nucl. Instr. \& Meth. In Phys. Res. 406(2) (1998) 177.

[9] A Q Zhou et al., Proc. of the 6th parti. acc. phys. symp. (Zhangjiajie, China), 1997, p.105.

[10] Saewoong Oh, Proc. of Hefei ICSRL (Hefei, China),
1989, p.111.

[11] L Wang et al., Proc. of the 6th parti. acc. phys. symp., 1997, p.156.

[12] L Wang et al., Proc. of the 2nd national symp. on acc. tech. (Ningbo, China), 1998, p.430.

[13] K J Fan et al., Proc. of the 6th particle acc. phys. symp. (Zhangjiajie, China), 1997, p.167.

[14] L Shang et al., Jour. of China Univ. of S. \& T. 28(2) (1998) 172.

[15] L Shang et al., High Power Laser and Particle Beams 29 (1) (1998) 103.

[16] L Shang et al., Proc. of the 2nd national symp. on acc. tech. (Ningbo, China), 1998, p.561.

[17] Y B Leng et al., Proc. of the 2nd national symp. on acc. tech. (Ningbo, China), 1998, p.299.

[18] K J Fan et al., Necl. Techniques, 21(3) (1998) 167.

[19] X Q Wang et al., High Energy Phys. \& Nucl. Phys. 22(8) (1998) 755.

[20] X Q Wang et al., Jour. of China Univ. of S. \& T. 28(2) (1998) 168. 